\title{
On The Use of Nonlinear Model Predictive Control for Pharmacological Therapy Optimization
}

\author{
Gabriele Pannocchia \\ Dipartimento di Ingegneria Chimica (DICCISM) \\ Univ. of Pisa. Pisa, Italy 56122 \\ Email: g.pannocchia@ing.unipi.it
}

\author{
Alberto Landi and Marco Laurino \\ Dipartimento di Sistemi Elettrici e Automazione \\ Univ. of Pisa. Pisa, Italy 56122 \\ Email: alberto.landi@dsea.unipi.it, Marco.Laurino@studenti.ing.unipi.it
}

\begin{abstract}
In this paper, we discuss the opportunities that Nonlinear Model Predictive Control (NMPC) offers in the area of pharmacological therapy as an effective way for achieving optimization goals such as drug usage reduction while respecting safety and therapeutic constraints imposed by physicians. We first review a general formulation of NMPC and discuss some issues associated with its application to biomedical processes. Next, we present two simulated biomedical applications of NMPC: the first one deals with the optimization of anti-retroviral therapy in HIV and the second one discusses the optimization of multiple injection insulin therapy in Type 1 diabetics. Both these examples serve to highlight the main advantages of NMPC.
\end{abstract}

\section{INTRODUCTION}

MPC algorithms use a (linear or nonlinear) model to forecast the system behavior over a future time horizon, and solve on-line optimization problems to determine, in real time, the optimal control policy [1]. Feedback from measurements is considered by updating the model predictions with suitable correction terms computed from the difference between the measured and predicted outputs. Such approaches have allowed a tremendous increase in profits, e.g. to refinery and petrochemical companies due to overall energy reduction usage, tighter quality control and throughput maximization [2].

The use of mathematical models and control algorithms in medicine represents nowadays a very active research area that embraces many different biomedical applications. The main goal of these research studies is to provide a systematic approach for the definition of therapeutic protocols by means of control algorithms similar to those commonly used in industrial contexts (e.g. chemical, refining, automotive, aerospace, etc.). The typical starting point in the development of such control algorithms is the derivation of a (dynamic) model of the system to be controlled. Such step requires, first of all, the definition of inputs (i.e. independent variables, which sometimes can be adjusted by physicians such as the drug dosage) and outputs (i.e. dependent variables, which can be possibly measured either continuously or more often with laboratory analysis). Then, such independent and dependent variables need to be "connected" by means mathematical relations (usually differential and/or algebraic equations), which contain a number of parameters. Due to popularity and reliability in many industrial applications, very often the use linear time-invariant deterministic models is attempted in biomedical applications as well. However, physiological systems are generally nonlinear, time variant and stochastic. Therefore, a trade off between model complexity and tractability is necessary in order to obtain a sufficiently accurate, yet manageable, system description.

We here describe the opportunities and issues associated to the application of MPC strategies in drug administration.

\section{A general Formulation OF NONLINEAR Model PREDICTIVE CONTROL}

MPC refers to control algorithms in which, at each decision time, a system model is used to forecast: an optimal "future" control sequence over a (finite) prediction horizon and an associated sequence of states originating from the current state value [1]. The control sequence is chosen in a way that an appropriate cost function, typically comprising a measure of the deviation of the future state/output sequence from reference target values and a measure of the control input effort, is minimized while state/output and control constraints are fulfilled. We here present a general formulation of Nonlinear Model Predictive Control (NMPC) with the aim of setting up the stage for the specific formulations used in two pharmacological therapy optimization applications discussed in Section III.

\section{A. Augmented model and state estimation}

We assume that a system model is available in discrete-time form $(k=0,1, \ldots$ is the discrete time index):

$$
\begin{aligned}
x(k+1) & =f(x(k), u(k))+w(k) \\
y(k) & =h(x(k))+v(k)
\end{aligned}
$$

in which $x \in \mathbb{R}^{n}$ is the state of the system, $u \in \mathbb{R}^{m}$ is the input (i.e. manipulated variable), $y \in \mathbb{R}^{p}$ is the output (i.e. measured variable); the independent variables $w \in \mathbb{R}^{n}$ and $v \in \mathbb{R}^{p}$ represent unpredictable disturbances, such as measurement noise and/or systematic model errors. The function $f: \mathbb{R}^{n} \times \mathbb{R}^{m} \rightarrow \mathbb{R}^{n}$ may be analytical or computed numerically from the integration of an underlying system of differential/algebraic equations. The function $h: \mathbb{R}^{n} \rightarrow \mathbb{R}^{p}$ is often a selection of the state $x$.

Since we only measure $y$ and given that $(w, v)$ may represent systematic errors (i.e. with nonzero mean), it is necessary to adopt an output feedback strategy to compute state estimates for the model (1) that are consistent with the 
input-output behavior of the actual (unknown) process. To this aim, we augment the system model (1) with fictitious disturbance variables [3], which lump the effect of systematic errors. The augmented system is:

$$
\begin{aligned}
\hat{x}(k+1) & =f(\hat{x}(k), u(k))+g_{x}(\hat{d}(k)) \\
\hat{d}(k+1) & =\hat{d}(k) \\
\hat{y}(k) & =h(\hat{x}(k))+g_{y}(\hat{d}(k))
\end{aligned}
$$

The vector $\hat{d} \in \mathbb{R}^{p}$ is the lumped disturbance assumed to have integral dynamics, $g_{x}: \mathbb{R}^{p} \rightarrow \mathbb{R}^{n}$ and $g_{y}: \mathbb{R}^{p} \rightarrow \mathbb{R}^{p}$ are appropriate (user-defined) functions that describe the effect of such lumped disturbance on the state and output evolution. Given the measured output value $y(k)$ and the augmented state predictions $(\hat{x}(k), \hat{d}(k))$, the prediction error is defined as:

$$
\epsilon(k)=y(k)-\hat{y}(k)=y(k)-\left(h(\hat{x}(k))+g_{y}(\hat{d}(k))\right)
$$

from which the augmented state predictions are updated:

$$
\begin{aligned}
& \hat{x}(k) \leftarrow \hat{x}(k)+l_{x}(\epsilon(k)) \\
& \hat{d}(k) \leftarrow \hat{d}(k)+l_{d}(\epsilon(k))
\end{aligned}
$$

in which $l_{x}: \mathbb{R}^{p} \rightarrow \mathbb{R}^{n}$ and $l_{d}: \mathbb{R}^{p} \rightarrow \mathbb{R}^{p}$ are suitable functions. Such updated estimates are used to predict the future state/output trajectories and to achieve output integral action (i.e. offset-free tracking) in the presence of systematic model errors, provided that the closed-loop system remains stable. Offset-free tracking occurs because $\hat{d}(k)$ changes at each sample time until the prediction error $\epsilon(k)$ vanishes, i.e. until the output prediction and the actual measurements become equal. Very often $\left(g_{x}, g_{y}, l_{x}, l_{d}\right)$, which define the socalled "disturbance model", are assumed to be linear [3], [4].

Sometimes, especially in biomedical applications, the output measurement is not available at each sample time. In such cases, we assume that the current prediction error is zero, and hence the update step (4) is not performed.

\section{B. Optimal control problem formulation}

We consider input and output constraints:

$$
u_{\min } \leq u(k) \leq u_{\max }, \quad y_{\min } \leq y(k) \leq y_{\max }
$$

Given a positive integer $N$, we define the following optimal control problem that is solved at each decision time $k$, given the current (updated) augmented state estimates $(\hat{x}(k), \hat{d}(k))$ :

$$
\min _{\left\{\tilde{u}_{i}\right\}_{i=0}^{N-1},\left\{\bar{e}_{i}, \underline{e}_{i}\right\}_{i=0}^{N}} \sum_{i=0}^{N-1} \ell\left(\tilde{y}_{i}, \tilde{u}_{i}, \bar{e}_{i}, \underline{e}_{i}\right)+\ell_{N}\left(\tilde{y}_{i}, \bar{e}_{i}, \underline{e}_{i}\right)
$$

subject to:

$$
\begin{gathered}
\tilde{x}_{0}=\hat{x}(k) \\
\tilde{x}_{i+1}=f\left(\tilde{x}_{i}, \tilde{u}_{i}\right)+g_{x}(\hat{d}(k)), \quad i=0, \ldots, N-1 \\
\tilde{y}_{i}=h\left(\tilde{x}_{i}\right)+g_{y}(\hat{d}(k)) \quad i=0, \ldots, N \\
u_{\min } \leq \tilde{u}_{i} \leq u_{\max } \quad i=0, \ldots, N-1 \\
y_{\min }-\underline{e}_{i} \leq \tilde{y}_{i} \leq y_{\max }+\bar{e}_{i} \quad i=0, \ldots, N \\
\underline{e}_{i} \geq 0, \quad \bar{e}_{i} \geq 0 \quad i=0, \ldots, N
\end{gathered}
$$

in which $\ell(\cdot)$ and $\ell_{N}(\cdot)$ are non-negative convex functions. The terms $\bar{e}_{i}$ and $\underline{e}_{i}$ represent violations of the output constraints (upper and lower, respectively), and such terms are penalized in the objective function to avoid large (if any) violations. Such approach, usually referred to as "soft-constraint" formulation [5], is in general necessary to ensure feasibility of the optimal control problem at all decision times.

The problem (6) is in general a nonlinear program (NLP), and must be solved with general purpose, often Sequential Quadratic Programming (SQP), algorithms [6]. One important observation is that NLP cannot be solved globally, i.e. the optimizer usually finds a local minimum rather than a global one. Furthermore, the computational time to solve an NLP could be relatively high. In fact, one important point that needs to be taken into account when considering MPC as possible control strategy is its computational cost (i.e. the CPU time) compared to the allowed decision time. Fortunately, this aspect is usually not crucial in decision support algorithms for pharmacological therapy optimization, in which the sample time is of the order of days/weeks.

\section{Closed-loop implementation}

Once the optimal input sequence, $\left\{\tilde{u}_{i}\right\}_{i=0}^{N-1}$, is computed from (6), only the first component is actually implemented:

$$
u(k)=\tilde{u}_{0}
$$

while the rest of the sequence is discarded. At the subsequent decision time, given the (updated) augmented state, a new optimal control sequence is computed. Such approach is usually referred to as "Receding Horizon Control" and it is the usual closed-loop implementation of MPC algorithms.

We can now describe the complete closed-loop implementation of the general NMPC algorithm.

Algorithm 1 Closed-loop implementation of NMPC

1) Given output $y(k)$ and predictions $(\hat{x}(k), \hat{d}(k))$, compute $\epsilon(k)$ from (3) and update $(\hat{x}(k), \hat{d}(k))$ from (4).

2) Solve the optimal control problem (6) and define the current input as in (7).

3) Forecast the next augmented state from (2).

4) Set $k \leftarrow k+1$ and go to 1 .

As discussed in Section III, some modifications may be necessary in each specific biomedical application.

\section{CASE STUdies}

We here present two simulated applications of NMPC to therapy protocol optimization problems.

\section{A. HIV control}

1) Preliminaries: In HIV infection, combination of drugs is used to reduce viral replication and to delay the progression of pathology [7], thus increasing life expectation and quality. In particular, Highly Active Anti-Retroviral Therapy (HAART) is a combination therapy that includes: 
- Reverse Transcriptase Inhibitors (RTI), to prevent cell-tocell transmission,

- Protease Inhibitors (PI), to prevent the production of virions by infected cells.

However, there are some limitations to the effectiveness of HAART. Infected cells have a short half-life (from days to months), but hidden reservoirs of virus contribute to an even slower disease phase, that makes complete eradication of the virus from the body impossible with current therapies. Because continued administration is associated with severe side effects and drug resistance generation, more recent research efforts have been directed at finding therapeutic protocols able to boost HIV-specific immune responses [8]. Some clinical data suggest that the so-called "Structured Treatment Interruptions" (STI) can boost immunity against HIV, especially when performed relatively early after infection [9]. The use of STI is currently a matter of debate (see [10]-[13] and references therein). We here present a novel approach towards the definition of an optimized STI protocol based on NMPC. Other details on this study can be found in [14].

2) Model and NMPC formulation: In the literature, several different models have been developed to describe the HIV evolution, and some review articles are already available [15], [16]. In this work we use a model recently developed by Landi et al. [17], described by six state variables:

$$
\left\{\begin{array}{l}
\dot{\mathbf{x}}=\lambda-d \mathbf{x}-\mathbf{r x v} \\
\dot{\mathbf{y}}=\mathbf{r x v}-a \mathbf{y}-p \mathbf{y z} \\
\dot{\mathbf{w}}=c \mathbf{x y \mathbf { w }}-c q \mathbf{y} \mathbf{w}-b \mathbf{w} \\
\dot{\mathbf{z}}=c q \mathbf{y} \mathbf{w}-h \mathbf{z} \\
\dot{\mathbf{v}}=k\left(1-\mu_{P} f_{P}\right) \mathbf{y}-u \mathbf{v} \\
\dot{\mathbf{r}}=r_{0}\left(1-\mu_{T} f_{T}\right)
\end{array}\right.
$$

The state variables (denoted in bold-face) are as follows: $\mathbf{x}$ and $\mathbf{y}$ are the concentration of healthy and infected CD4+ cells, respectively; $\mathbf{w}$ and $\mathbf{z}$ are the concentration of Cytotoxic T-Lymphocyte precursors and effectors, respectively; $\mathbf{v}$ is the concentration of free virions and $\mathbf{r}$ represents the virus aggressiveness. The input variables are $f_{P}$ and $f_{T}$, which represent the normalized uptake of RPI and RTI drugs. This model differs from other HIV models described in the literature, in the introduction of the new state variable $\mathbf{r}$. According to the equation describing the $\mathbf{r}$-state dynamics, $\mathbf{r}$ increases linearly with time in the case of an untreated HIV-infected individual, with a growth rate that depends on the constant $r_{0}$. Notice that the considered model does not exhibit any stable (immune) steady state, given the fact that we will assume that the model parameters are chosen such that $\left(\mu_{T} f_{T}\right)<1$, and hence $\dot{\mathbf{r}}>0$, i.e. the virulence never becomes a constant.

Consistently with medical practice, we assume to measure only two state variables, $(\mathbf{x}, \log \mathbf{v})$, from which the output function $h(\cdot)$ is accordingly defined. Furthermore, we consider a discretization time of one week, from which the state evolution function $f(\cdot)$ is computed via numerical integration. In the augmented model (2), we consider $g_{x}=0$ and $g_{y}=\hat{d}(k)$, and the updating functions in (4) are $l_{x}=0$ and $l_{d}=\epsilon(k)$.
Notice that the update step (4) is performed only every four weeks, i.e. when the output measurements are available.

At each decision time, i.e. every week, we solve the NMPC problem (6), with horizon of $N=8$ and with some specific modifications as detailed. Only a lower bound on the first output $(\mathbf{x})$ and an upper bound on the second output $(\log \mathbf{v})$ are considered. Consequently, the violations $\underline{e}$ and $\bar{e}$ exist only for these two output constraints. The inputs $u=\left[f_{P}, f_{T}\right]^{\prime}$ are constrained to be equal, and either 0 or 1 . The cost functions are linear and defined as follows:

$$
\ell(\cdot)=c_{P} f_{P}+c_{T} f_{T}+q_{x} \underline{e}+q_{v} \bar{e}, \quad \ell_{N}(\cdot)=q_{x} \underline{e}+q_{v} \bar{e}
$$

in which all parameters are positive and described elsewhere [14]. We observe that the solution of the NMPC problem with the addition of the binary constraint on the inputs requires, in principle, a mixed-integer nonlinear programming solver. However, we used a full enumeration approach in which the cost of all possible sequences of $N$ weeks, each at either full or zero therapy, is computed. Then, the sequence with least cost is chosen as the optimal one, and such approach allows us to compute the global minimum. The required CPU time, using Matlab, is of the order of 20-30 seconds, thus perfectly admissible compared to the discretization time.

3) Results and discussion: In our simulation study, we consider three different reference patients obtained by using slightly different model parameters. The different dynamic response of these patients under a full (i.e. sustained) HAART started 2 months after the infection is depicted in Fig. 1 over a period of two years. We notice that Patient A shows the fastest disease progression, whereas Patient $\mathrm{C}$ shows the slowest progression, and Patient B shows an intermediate behavior. It can be noticed that the viral load shows faster dynamics compared to the CD4+ concentration, and furthermore during the first one-two months from infection, a natural immunological rebound of CD4+ concentration and reduction of viral load occur even without therapy. In order to assess the achieved performance, we use three indicators: (i) overall drug consumption (expressed in weeks of full HAART), (ii) average CD4+ concentration, (iii) average viral load.

We present in Fig. 2 the simulated response of Patient B using NMPC-based therapy protocol, assuming perfect knowledge of the patient behavior and noise-free measurements. In this nominal situation, it follows that $\epsilon(k)=0$ at all times, and so $\hat{d}(k)=0$. We consider a lower bound for CD4+ of 800 cells $/ \mathrm{mm}^{3}$ and an upper bound on the viral load of 300 copies $/ \mathrm{ml}$. The therapy is started two months after the infection. As expected, the NMPC-based therapy protocol is able to satisfy the constraints on CD4+ concentration and on the viral load while reducing the drug dosage with respect to sustained HAART therapy at maximum dosage. In particular, the induced Structured Treatment Interruption protocol comprises 77 weeks on and 19 weeks off.

We also tested the closed-loop response of Patient B in other situations, namely when the therapy is started later (4 months after infection) or earlier (1 month after infection), and with noisy measurements (noise-to-signal ratio of $10 \%$ ). The 

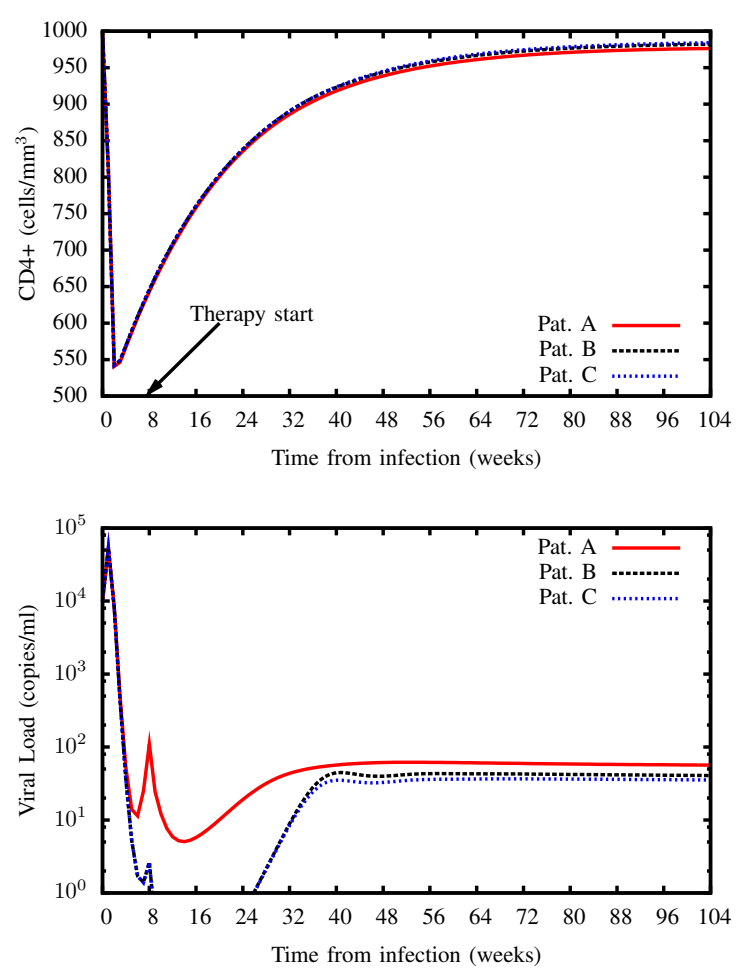

Fig. 1. Patients response under HAART started after 2 months from infection over a period of two years.

corresponding results are summarized in Table I by reporting the performance indicators. From these results, we can observe that the proposed NMPC-based protocol does not suffer from sensitivity to noise and can take easily into account variations in the starting time. In fact, in all cases the average value of CD4+ is significantly above the lower limit and the viral load is kept below its upper bound without the necessity of full HAART. In this way, the severe side effects of a full therapy can be minimized without jeopardizing the disease control.

In order to assess the robustness of the proposed NMPC strategy with respect to model errors, we tested the control of Patients A and C using NMPC algorithms based on the model of Patient B. In such cases, there is inherent mismatch between the model prediction and the patient response, and such mismatch is "captured" into the prediction error $\epsilon(k)$ every four weeks when the measurements of CD4+ and viral load become available. In the sake of space, results are only presented in Table I from which we observe again the ability of the NMPC protocols to satisfy the therapeutic constraints, despite the fact that an incorrect model is used. It is interesting to notice that an STI protocol is essentially inappropriate for Patient A (the one with fastest disease progression) as only 1 week off is computed. On the other hand, for Patient $\mathrm{C}$ (the one with slowest disease progression) the STI protocol comprises 56 weeks on and 40 weeks off. From these results, it is clear that the NMPC-based protocols are adjusted to the specific, yet unknown, characteristics of the controlled patient.
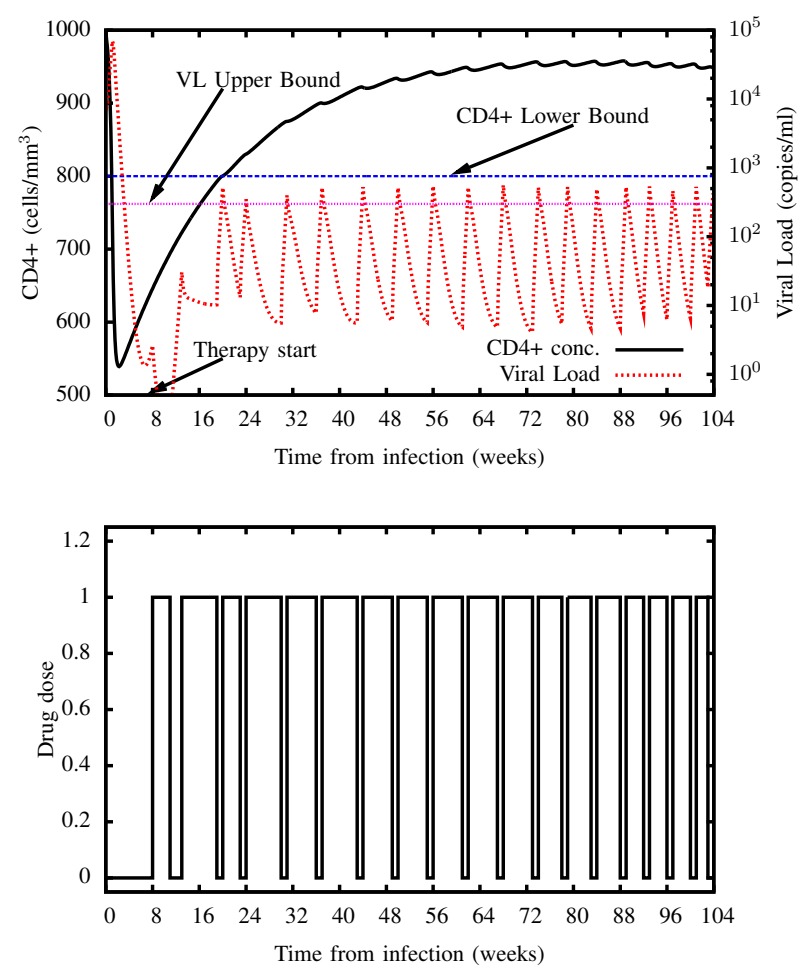

Fig. 2. Closed-loop response of Pat. B with NMPC. Top plot: CD4+ concentration (left axis) and viral load (right axis), bottom plot: drug uptake.

TABLE I

DRUG CONSUMPTION (WEEKS OF FULL THERAPY), AVER. CD4+ (cell $/ \mathrm{mm}^{3}$ ) AND AVER. VIRAL LOAD (COPIES/ML).

(Patient B)

\begin{tabular}{lccc}
\hline Case & Drug & $\overline{\mathrm{CD} 4+}$ & $\overline{\mathrm{VL}}$ \\
\hline No treatment & 0 & 726 & 399 \\
Full HAART & 96 & 897 & 29.4 \\
NMPC & 77 & 881 & 32.6 \\
NMPC started after $4 \mathrm{~m}$. & 64 & 861 & 54.7 \\
NMPC started after 1 m. & 78 & 881 & 29.8 \\
NMPC noise measurements (10\%) & 74 & 877 & 40.6 \\
\hline
\end{tabular}

\begin{tabular}{lccc}
\multicolumn{4}{c}{ (Patient A) } \\
\\
\hline No treatment & 0 & 676 & 628 \\
Full HAART & 96 & 893 & 48.8 \\
NMPC based on pat.B & 95 & 891 & 51.7 \\
\hline
\end{tabular}

\begin{tabular}{lccc}
\multicolumn{4}{c}{ (Patient C) } \\
\hline No treatment & 0 & 741 & 362 \\
Full HAART & 96 & 899 & 26.2 \\
NMPC based on pat.B & 56 & 860 & 73.2 \\
\hline
\end{tabular}

\section{B. Type I Diabetes Mellitus control}

1) Introduction: Type I diabetes mellitus is a metabolic disease in which an absolute deficiency of insulin secretion causes hyperglycemia, which is known to be source of several long-term complications, including heart disease and stroke, hypertension, retinopathy, nephropathy, and neuropathy. In order to achieve euglycemic control, patients depend on the 
supply of external insulin, which is typically administrated by means of multiple daily injections.

A significant amount of research studies aim at implementing the so-called "artificial pancreas", a closed-loop system comprising: an implantable glucose sensor, an insulin delivery pump (and a reservoir), and a control algorithm that adjusts the insulin rate in order to maintain a normal glycemic level, in spite of disturbances (e.g. meals and physical activity). See [18], [19] and references therein for a current assessment. In this respect, the main technological issues are associated to the availability of reliable implantable sensors and to reducing dimensions and portability of the insulin pumps.

Still, the majority of diabetics is currently treated with multiple injections, and the objective of the present study is to define a Nonlinear MPC based therapeutic protocol optimization strategy to determine the appropriate insulin doses for each specific patient. We consider to optimize a protocol comprising three pre-prendial injections of Lispro insulin and an injection of Glargine insulin for overnight control.

2) Physiological model and NMPC algorithm: Many physiological models for the glucose/insulin system have been published in the literature. In this work we use the compartmental model developed by Lehmann and Deutsch [20], chosen because it is particularly suitable for simulation of multiple daily injection protocols comprising different insulin preparations. The model differential equations are (notice that state variables are shown in bold-face):

$$
\left\{\begin{aligned}
\dot{\mathbf{I}} & =\frac{I_{a b s}(t)}{V_{I}}-k_{e} \mathbf{I} \\
\dot{\mathbf{I}} & =k_{1} \mathbf{I}-k_{2} \mathbf{I}_{a} \\
\dot{\mathbf{G}} & =\frac{k_{a b s} \mathbf{G}_{g u t}+G_{N H B}(t)-G_{\text {out }}(t)-G_{r e n}(t)}{V_{G}} \\
\mathbf{G}_{\text {gut }} & =G_{\text {empt }}(t)-k_{a b s} \mathbf{G}_{\text {gut }}
\end{aligned}\right.
$$

in which $\mathbf{I}$ and $\mathbf{I}_{a}$ are the plasmatic and active insulin concentration, respectively; $\mathbf{G}$ and $\mathbf{G}_{\text {gut }}$ are the plasmatic and gut glucose concentration, respectively. The time varying function $I_{a b s}(t)$ is the insulin absorbed with the previous injections and the contribution of each injection can be expressed as:

$$
I_{a b s}(t)=\frac{s\left(t-t_{0}\right)^{s-1} T_{50}^{s} D}{\left(T_{50}^{s}+\left(t-t_{0}\right)^{s}\right)^{2}}
$$

in which $t_{0}$ is the injection time, $D$ is the injected dose, while the parameters $s$ and $T_{50}$ vary with the insulin type (normal, NPH, etc.). $G_{e m p t}(t)$ represents the glucose gastric emptying rate occurring after a meal and has trapezoidal (or triangular) shape. $G_{N H B}(t)$ is the so-called "Net Hepatic Glucose Balance" and depends on the active insulin $\mathbf{I}_{a}$ and on the glucose level G. $G_{\text {out }}(t)$ represents the peripheral use of glucose and also depends on $\mathbf{I}_{a}$ and G. Finally, $G_{r e n}(t)$ is the glucose renal excretion and depends on $\mathbf{G}$. For a complete description the interested reader is referred to [20], [21].

In this study, we consider a therapy protocol comprising three daily injections of Lispro insulin (right before breakfast, lunch and dinner), and one injection of Glargine administrated before bed time. These two recent insulin types were not considered in the original model and, therefore, we evaluated the associated parameters from data as discussed in [21]. Thus, the overall nonlinear model has two manipulated inputs, the doses of Glargine and Lispro injected, and external "disturbances" associated with the carbohydrate uptakes.

We assume to measure the glucose level in the morning, one hour before breakfast, and this measurement is used to update the augmented state predictions from (4). As in the previous application we use the following disturbance model: $g_{x}=$ $0, g_{y}=\hat{d}(k)$ and $l_{x}=0, l_{d}=\epsilon(k)$. In the optimal control problem (6), we assume to know in advance the (predicted) amount carbohydrates contained in the meals, and we consider quadratic stage cost functions:

$$
\begin{aligned}
\ell(\cdot) & =q\left(\tilde{y}_{i}-y_{s}\right)^{2}+s\left(\tilde{u}_{i}-u_{i}^{s}\right)^{2}+\bar{q} \bar{e}_{i}^{2}+\underline{q}_{i}^{2} \\
\ell_{N}(\cdot) & =q\left(\tilde{y}_{i}-y_{s}\right)^{2}+\bar{q} \bar{e}_{i}^{2}+\underline{q} \underline{e}_{i}^{2}
\end{aligned}
$$

$\left(\tilde{y}_{i}, \tilde{u}_{i}\right)$ are the predicted glucose concentration and insulin dose at time $i$, respectively; $y_{s}$ is the euglycemic level; $u_{i}^{s}$ is the dose predicted in the previous NMPC problem. The stage cost is evaluated every 15 minutes for a horizon of 3 days.

3) Results and comments: We consider the application of the proposed NMPC algorithm to a reference (female) patient whose model parameters can be found in [20], [22]. In reference conditions, we consider the following values for the carbohydrates contained in the meals: $20 \mathrm{~g}$ (8:00 $\mathrm{AM}$, breakfast), $30 \mathrm{~g}$ (10:00 AM, snack), $70 \mathrm{~g}$ (1:00 PM, lunch), $20 \mathrm{~g}$ (5:00 PM, snack), $70 \mathrm{~g}$ (8:00 PM, dinner). The following reference protocol is considered for the first day: $2 \mathrm{U}$ Lispro at 8:00 AM, $4 \mathrm{U}$ Lispro at 1:00 PM, 4 Lispro at 8:00 PM, $10 \mathrm{U}$ Glargine at 10:30 PM. For subsequent days, the daily protocol is computed by the NMPC algorithm given the glucose concentration measurement made at 7:00 AM. The reference glycemic level is $y_{s}=7.5 \mathrm{mmol} / \mathrm{L}$, the hyperglycemic and hypoglycemic bounds are $y_{\max }=$ $10 \mathrm{mmol} / \mathrm{L}$ and $y_{\min }=4 \mathrm{mmol} / \mathrm{L}$, respectively. The cost function weights are: $q=1, s=0.01, \bar{q}=100, q=10^{3}$. A dedicated nonlinear "trust region" optimization [6] algorithm was written in Octave/C++, and a randomized procedure for the computation of the initial guess was accurately developed to reduce local minima effects.

We present in Fig. 3 the glucose concentration profile and the insulin doses administrated over a four day period. In this first simulation the model used by the NMPC algorithm is perfect. We can immediately notice that the initial reference protocol is too mild. In fact, during the first day, the average glycemic level is $9.6 \mathrm{mmol} / \mathrm{L}$ and the hyperglycemic bound is exceeded, during the whole day, for 4 more hours. On the other hand, when the protocol is optimized by the NMPC algorithm, the glycemic level is always within the bounds, with an average level of $7.52 \mathrm{mmol} / \mathrm{L}$. We next present in Fig. 4 the corresponding results obtained when the model used by NMPC is not perfect (the two main patient dependent parameters, the Hepatic and Peripheral sensitivities are incorrect). Again, we can notice that the NMPC algorithm is able to lower the average glucose concentration (to a level 

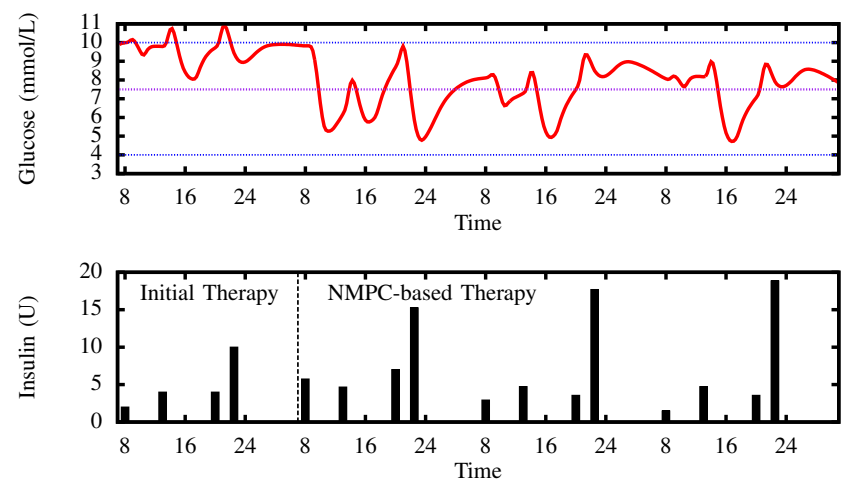

Fig. 3. Glucose concentration and insulin doses during a four day period. NMPC based on exact model is used from the second day.
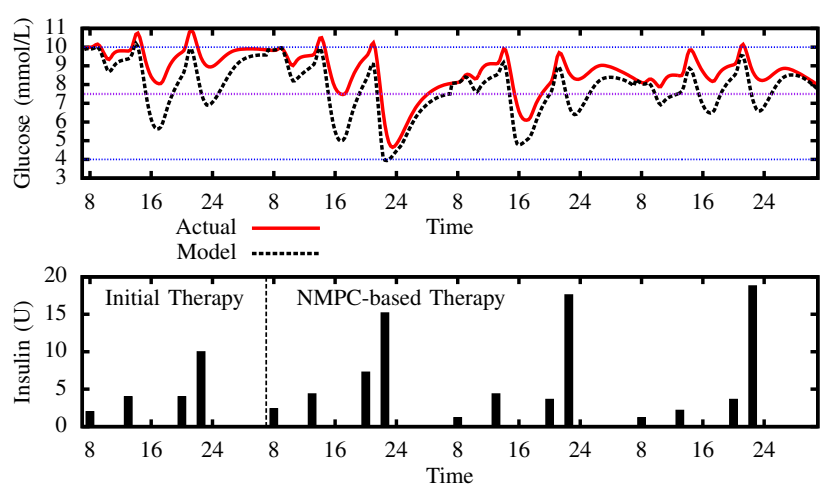

Fig. 4. Glucose concentration, model prediction and insulin doses. NMPC based on incorrect model is used from the second day.

of $8.4 \mathrm{mmol} / \mathrm{L}$ ). Furthermore, it is interesting to notice the effect of feedback from the morning glucose measurements. During Day 2 (the first one using NMPC) there are still some hyperglycemic episodes (overall duration of $1.5 \mathrm{hr}$ ), whereas during the remaining two days only $0.5 \mathrm{hr}$ of hyperglycemic conditions are experienced. This is due to the fact that the updated model predictions become more accurate as feedback measurements are acquired. We also simulated the case in which the amounts of carbohydrates actually assumed during the meals are different from the values known by the NMPC algorithm, and also in this case (whose results are not shown due to space limitations) the algorithm shows remarkable robustness properties.

\section{Conclusions}

In this paper we presented a general framework of Nonlinear Model Predictive Control (NMPC) strategies and two simulated applications to drug administration problems. We showed how such NMPC strategies are well suited for this type of biomedical "systems" due to their definite applicability to the case of infrequent measurements and the presence of constraints on both decision and controlled variables (where other non-predictive feedback algorithm can be hardly applied). Furthermore, the adopted feedback mechanism allows one to embed effective robustness properties, and therefore to cope with unavoidable model errors.

\section{ACKNOWLEDGMENT}

We acknowledge M. Merolla, I. Fastelli, A. Lo Biondo and G. Mancuso for preliminary works on MPC applications to diabetes control.

\section{REFERENCES}

[1] D. Q. Mayne, J. B. Rawlings, C. V. Rao, and P. O. M. Scokaert, "Constrained model predictive control: stability and optimality," Automatica, vol. 36, pp. 789-814, 2000.

[2] S. J. Qin and T. A. Badgwell, "A survey of industrial model predictive control technology," Cont. Eng. Pract., vol. 11, pp. 733-764, 2003.

[3] G. Pannocchia and J. B. Rawlings, "Disturbance models for offset-free model predictive control," AIChE J., vol. 49, pp. 426-437, 2003.

[4] G. Pannocchia and A. Bemporad, "Combined design of disturbance model and observer for offset-free model predictive control," IEEE Trans. Auto. Cont., vol. 52, no. 6, pp. 1048-1053, 2007.

[5] P. O. M. Scokaert and J. B. Rawlings, "Feasibility issues in linear model predictive control," AIChE J., vol. 45, pp. 1649-1659, 1999.

[6] J. Nocedal and S. J. Wright, Numerical Optimization. Springer, 1999.

[7] B. M. Adams and et al., "HIV dynamics: modeling, data analysis, and optimal treatment protocols," J. of Comput. \& Applied Math., vol. 184, pp. 10-49, 2005.

[8] D. Wodarz, K. M. Page, R. Arnaout, A. R. Thomsen, J. D. Lifson, and M. A. Nowak, "A new theory of cytotoxic T-lymphocyte memory: Implications for HIV treatment," Philos. Trans. R. Soc. Lond. B Biol. Sci., vol. 355, pp. 329-343, 2000.

[9] D. Wodarz and M. A. Nowak, "Specific therapy regimes could lead to long-term immunological control of HIV," Proc. Natl. Acad. Sci. USA, vol. 96, pp. 14 464-14 469, 1999.

[10] D. E. Kaufmann, M. Lichterfeld, M. A. M. M. Addo, M. N. Johnston, P. K. Lee, B. S. Wagner, E. T. Kalife, D. Strick, E. S. Rosenberg, and B. D. Walker, "Limited durability of viral control following treated acute HIV infection," PLos Med., vol. 1, no. 2, pp. 137-148, 2004.

[11] R. Zurakowski and A. R. Teel, "A model predictive control based scheduling method for HIV therapy," J. Theoretical Biology, vol. 238, pp. 368-382, 2006.

[12] J. H. Ko, W. H. Kim, and C. C. Chung, "Optimized structured treatment interruption for HIV therapy and its performance analysis on controllability," IEEE Trans. Biom. Eng., vol. 53, no. 3, pp. 380-386, 2006.

[13] A. M. Jeffrey, X. Xia, and I. K. Craig, "IV. Structured treatment interruptions: A control mathematical approach to protocol design," $J$. Process Control, vol. 17, pp. 586-590, 2007.

[14] G. Pannocchia, M. Laurino, and A. Landi, "A model predictive control strategy towards optimal structured treatment interruptions in anti-HIV therapy," December 2009, accepted for publication in IEEE Transactions on Biomedical Engineering.

[15] D. Wodarz and M. A. Nowak, "Mathematical models of HIV pathogenesis and treatment," BioEssays, vol. 24, pp. 1178-1187, 2002.

[16] X. Xia, "Modelling of HIV infection: Vaccine readiness, drug effectiveness and therapeutical failures," J. Process Control, vol. 17, pp. 253-260, 2007.

[17] A. Landi, A. Mazzoldi, A. Chiara, M. Bianchi, A. Cavallini, M. Laurino, L. Ricotti, R. Iuliano, B. Matteoli, and L. Ceccherini-Nelli, "Modelling and control of HIV dynamics," Comput. Methods Programs Biomed., vol. 89, no. 2, pp. 162-168, 2008.

[18] B. W. Bequette, "A critical assessment of algorithms and challanges in the development of a closed-loop artificial pancreas," Diabetes Technology \& Therapeutics, vol. 7, pp. 28-47, 2005.

[19] L. Magni, D. M. Raimondo, L. Bossi, C. Dalla Man, G. De Nicolao, B. Kovatchev, and C. Cobelli, "Model predictive control of type 1 diabetes: An in silico trial," J. Diab. Sci. Tech., vol. 1, pp. 804-812, 2007.

[20] E. D. Lehmann and T. Deutsch, "A physiological model for glucoseinsulin interaction in type I diabetes mellitus," Journal of Biomedical Engineering, vol. 14, pp. 235-242, 1992.

[21] I. Fastelli, "Development of predictive algorithms for optimization of the intensive insulin therapy (in italian)," Master's thesis, Chemical Engineering - Univ. of Pisa, 2005.

[22] E. D. Lehmann, "Preliminary experience with the internet release of aida - an interactive educational diabetes simulator," Comp. Meth. \& Progr. in Biomedicine, vol. 56, pp. 109-132, 1998. 